Исполняемое моделирование бизнес-процессов

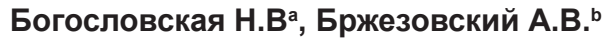

ФГАОУ ВО «Санкт-Петербургский государственный университет аэрокосмического приборостроения»,

Санкт-Петербург, Российская Федерация.

a iD ORCID: https://orcid.org/0000-0003-1897-0577, e-mail: nvbogoslov@mail.ru

b iD ORCID: https://orcid.org/0000-0002-5750-2664, e-mail: avb@aanet.ru

Резюме: Как отмечается в пояснительной записке к профессиональному стандарту «Специалист по информационным системам», успех внедрения информационных систем в значительной степени определяется точностью их адаптации к бизнес-процессам организации-заказчика. В качестве техники извлечения требований к информационным системам в настоящее время часто используется нотация BPMN. B BPMN предусмотрена исполняемая семантика, позволяющая не просто составить детальную спецификацию бизнес-процесса, но и произвести его модельное выполнение с получением численных оценок. Цель: выбрать инструментальные средства для обучения студентов, позволяющие реализовать третий уровень моделирования, из числа предусмотренных нотацией BPMN: описательный, аналитический и исполняемый. Определить методику преподавания бизнес-моделирования, учитывающую как возможность исполнения бизнес-процессов в универсальных средах исполнения BPMN, так и в виде надстройки над конфигурацией информационной системы. Методы: сравнительный анализ и тестирование функциональных возможностей, предоставляемых для разработки бизнес-процессов в инструментальных средах Bizagi и 1C:Предприятие, с учетом создания необходимых для исполнения BPMN слоев данных и представления. Результаты. Сформулированы основные этапы бизнес-моделирования, проведено функциональное сравнение инструментальных средств для работы на этапах: разработки модели бизнес-процесса; разработки слоя данных, сопровождающих выполнение процесса; отображения данных на задачи процесса - фрормы пользовательских задач; программирования бизнес-правил, регулирующих выполнение процесса; исполнения бизнес-процесса в разрезе пользователей-исполнителей. Особенностью $1 \mathrm{C:Предприятие} \mathrm{является} \mathrm{то,} \mathrm{что} \mathrm{бизнес-процесс} \mathrm{является} \mathrm{по}$ сути надстройкой над слоями данных и представлений готовой конфигурации процессно-ориентированной инфрормационной системы. Авторы считают, что и один, и второй подход заслуживает внимания с точки зрения учебного процесса в вузе для студентов специальности 09.03.02 «Информационные системы и технологии».

Ключевые слова: нотация и модель бизнес-процессов, исполняемая семантика BPMN, инструментальные средства для разработки и исполнения моделей BPMN.

Для цитирования: Богословская Н.В. Исполняемое моделирование бизнес-процессов / Н.В. Богословская, А.В. Бржезовский // Развитие образования. - 2020. - № 1 (7). - С. 29-34. DOI:10.31483/r-74738.

\section{Executable Business Process Modeling}

\author{
Natalya V. Bogoslovskayaa ${ }^{a}$ Aleksandr V. Brzhezovskii \\ FSAEI of HE "Saint Petersburg State University of Aerospace Instrumentation", \\ Saint Petersburg, Russian Federation. \\ DORCID: https://orcid.org/0000-0003-1897-0577, e-mail: nvbogoslov@mail.ru \\ b(iDORCID: https://orcid.org/0000-0002-5750-2664, e-mail: avb@aanet.ru
}

\begin{abstract}
As noted in the explanatory note to the «Information Systems Specialist» professional standard, the success of implementing information systems is largely determined by the accuracy of their adaptation to the business processes of the customer organization. Today, BPMN notation is often used as a tool for extracting requirements for information systems. BPMN provides executable semantics that make it possible not only to draw up a detailed specification of a business process, but also to model it with numerical estimates. The aims of this study are to choose tools for teaching students from the number provided by BPMN notation: descriptive, analytical and executable, which allow to implement the third level of modeling; to define a methodology for teaching business modeling, including the ability to execute business processes in the universal BPMN runtimes and as an add-on on the configuration of the information system. The following methods have been used: comparative analysis and testing of the functionality provided for the development of business processes in the Bizagi and 1C: Enterprise tool environments, including the creation of the data and presentation layers necessary for BPMN execution. As a result, the main stages of business modeling are formulated, a functional comparison of tools for working at the following stages has been carried out: development of a business process model; development of a data layer that supports the execution of the process; mapping data to process tasks - forms of user tasks; programming business rules governing the process; execution of a business process by user-performer. The peculiarity of 1C: Enterprise is that the business process is essentially a superstructure over the layers of data and representations of the finished configuration of a process-oriented information system. The authors believe that both the first and the second approach deserve attention in terms of the educational process at the university for students of 09.03 .02 «Information systems and technologies» specialty.
\end{abstract}

Keywords: Business Process Model and Notation, BPMN Execution Semantics, tools for developing and executing BPMN models.

For citation: Natalya V. Bogoslovskaya, \& Aleksandr V. Brzhezovskii (2020). Executable Business Process Modeling. Razvitie obrazovaniya = Development of education, 1(7), 29-34. (In Russ.) DOI:10.31483/r-74738. 


\section{Алгебрӑпа геометри вӗренӳ кӗнекисен ӗс̧левлӗх задачисене ТПВ ФГОСӗн требованийӗсене тӗпе хурса тишкерни}

Богословская Н.B ${ }^{\text {, Бржезовский А.B. }}{ }^{\text {- }}$

АВ ФПА «Санкт-Петербургӑн аэрокосмос приборостроенийӗн патшалӑх университечӗ» ВУ, Санкт-Петербург, Рас̧с̧ей Патшалӑхе̌.

a ID ORCID: https://orcid.org/0000-0003-1897-0577, e-mail: nvbogoslov@mail.ru

b(iD ORCID: https://orcid.org/0000-0002-5750-2664, e-mail: avb@aanet.ru

Аннотаци: «Информаци системипе ӗс̧лекен специалист» професси стандарчӗн ӑнлантару с̧ырӑвӗнче каланӑ тӑрӑх, информаци системисене пурнӑс̧а кӗртнин ӑнӑс̧ӑвӗ ытларах чухне заказчик организацин бизнес процесӗсене туллин хӑнӑхнинчен килет. Информаци системисем мӗнле пулмаллине ус̧ӑмлатакан требованисене кӑларса илмелли техника вырӑнне хальхи вӑхӑтра BPMN нотаципе усӑ курас̧с̧ӗ. BPMNра пурнӑс̧ланакан семантикӑна вырнас̧тарнӑ. Вӑл бизнес процеса тӗплӗн спецификациленисӗр пус̧не ӑна числоллӑ хаклав туса модельлӗ пурнӑс̧лама май парать. Тӗллев: студентсене вӗрентме юрӑхлӑ моделированин BPMN нотацире палӑртнисенчен вис̧ӗме̌ш шайне с̧итме май паракан хатӗр-хӗтӗр (сӑнламалли, тишкермелли, пурнӑс̧ламалли) янтӑласси. Бизнес моделированине вӗрентмелли методикӑна тупасси. Вӑл бизнес процесӗсене яланхи условисенче хута ярса суул ус̧нисӗр пус̧не инфрормаци системин конфигурацийӗ с̧ийӗн тӑракан хатӗр пулса тӑмалла. Меслетсем: бизнес процесне функци пултараслӑхне танлаштарса тишкерни тата тестлани. Тишкерме тата тестлама Bizagi тата 1C:Предпирияти инструмент талккӑшӗ май парать. Результатсем: бизнеса модельлемелли тӗп тапхӑрсене тупса палӑртнӑ; с̧ав тапхӑрсенче (бизнес процесӑн ӗлкине хатерлесси; даннӑйсен процеса тумалли сийне хатӗрлесси т. ыт. те) усӑ курмалли инструментарие танлаштарнӑ. 1C:Предпириятин уйрӑмлӑхӗ акӑ мӗн: бизнес процесӗ - даннӑйсем с̧ийӗн, информаци системин хатӗр конфигураци с̧ийӗн янтӑланакан пулӑм пулса тӑрать. Авторсем акӑ мӗн шухӑшлас̧с̧ӗ: тупса палартнӑ суул-йӗртен вуз вӗренӗвӗнче студентсене 09.03.02 «Информаци системисемпе технологийӗсем» специальнос̧па хатерленӗ май иккӗшне те шута илмелле.

Tӗп сӑмахсем: бизнес процесӗсен нотацийӗ тата ӗлки (моделӗ), BPMNра пурнӑс̧ланакан семантика, BPMN моделӗсене хатӗрлесе вӗсемпе пурнӑс̧ра усӑ курмалли инструментсем.

Цитатӑлама: Богословская Н.В. Бизнес процесӗсене пурнӑс̧ламалла модельлени / Н.В. Богословская, А.В. Бржезовский // Вӗренў аталанӑвӗ. - 2020. - № 1 (7) . - C. 29-34. DOI:10.31483/r-74738.

\section{Введение}

$\Pi$ риведение образовательной программы в соответствие с федеральным государственным образовательным стандартом высшего образования - бакалавриат по направлению подготовки 09.03.02 «Информационные системы и технологии» потребовало существенного пересмотра профессиональных компетенций. Основным отличием нового образовательного стандарта является учет требований профессионального стандарта «Специалист по информационным системам» и др. из данной группы.

В перечне трудовых функций профессионального стандарта 06.015 указаны следующие виды деятельности [1]:

- В/06.5 - адаптация бизнес-процессов заказчика к возможностям типовой ИС;

- В/19.5 - интеграция ИС с существующими ИС заказчика;

- С/08.6 - разработка модели бизнес-процессов заказчика;

- C/09.6 - адаптация бизнес-процессов заказчика к возможностям ИС;

- D/08.7 - разработка инструментов и методов проектирования бизнес-процессов заказчика.

В сложившейся практике преподавания технологий бизнес-моделирования авторы делали основной упор на изучении современного стандарта моделирования бизнес-процессов BPMN (Business Process Model and Notation) и использовании программных средств моделирования [2], что соответствовало первым двум уровням в нотации BPMN [3, с. 2]:

1. Описательное моделирование (descriptive modeling) - простое моделирование, описывающее процесс на бизнес-уровне с использованием базовых элементов нотации.

2. Аналитическое моделирование (analytical modeling) - моделирование, включающее альтернативные пути и исключения, требующее описывать процесс с максимально необходимой детализацией.

3. Исполняемое моделирование (executable modeling) - моделирование процесса, пригодного для исполнения в BPMS/BPMT (Business Process Management System/Tool - система (инструмент) управления бизнес-процессами). Разработка исполняемых моделей требует добавления в модель дополнительных атрибутов и параметров, а также высокой квалификация разработчика.

Третий уровень - исполняемое моделирование не рассматривался в учебном процессе, так как для его реализации требуется среда исполнения. В качестве среды исполнения бизнес-процессов можно использовать систему управления бизнес-процессами BPMS/BPMT или процессно-ориентированную информационную систему, которая поддерживает бизнес-процессы.

В качестве системы BPMS/BPMT была выбрана Bizagi Studio - это ВРМ-система, разработанная одноименной компанией, которая предлагает использовать платформу для быстрой автоматизации процессов любого рода через механизмы «drag and drop», способную учитывать персонализированный контекстный опыт разработчиков [4]. В качестве варианта процессно-ориентированной системы была выбрана платформа 1C:Предприятие 8.3, где «механизм бизнес-процессов это один из прикладных механизмов платформы. Он позволяет описывать, создавать и управлять выполнением бизнес-процессов в прикладных решениях» [5]. 


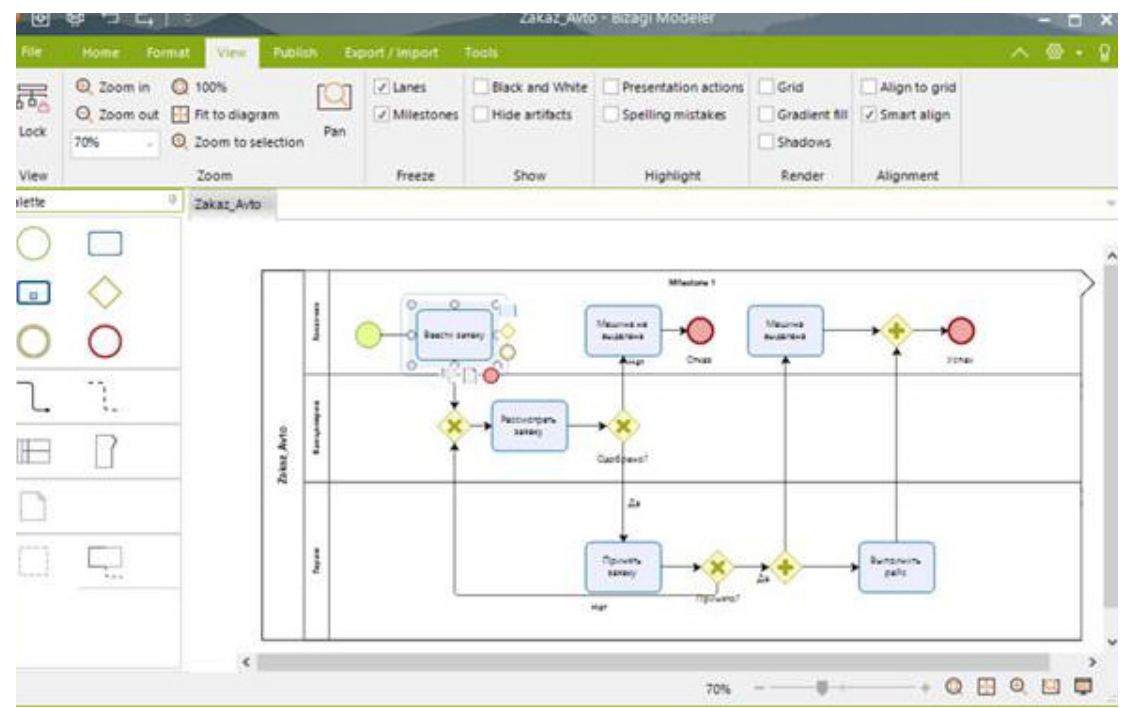

Рис. 1. Бизнес-процесс в 1C

Fig. 1. Business process in $1 C$

И одна, и другая системы поддерживают нотацию моделирования бизнес-процессов BPMN, что позволяет сравнить особенности создания и исполнения моделей в различных средах.

Благодаря тому, что модельможет быть исполнена как в среде управления бизнес-процессами, так и в среде реальной автоматизированной системы, студенты имеют возможность наглядно увидеть все аспекты процессно-ориентированной технологии: разработку модели бизнес-процесса (различие в инструментарии), создание и использование слоя данных, проектирование форм для задач бизнес-процесса, описание бизнес-правил, запуск и тестирование бизнес-процесса, получение метрик процесса.

Разработка и исполнение

бизнес-проиессов в системе 1С:Предприятие

Основными объектами конфигурации в системе 1С:Предприятие, реализующими моделирование и исполнение бизнес-процесса, являются прикладные объекты: Бизнес-процесс, Задача, Регистры сведений. Отличительной особенностью работы в 1С является использование технологии метаданных - визуальное конструирование прикладного решения. Разработчик с помощью диалоговых средства добавляет новый объект конфигурации в прикладное решение и автоматически получает определения ся из элементов, являющихся подмножеством BPMN. Задачи в модели

97 Текущие вызовы: 0 Накопленные вызовы: 37 соответствуют пользовательским заданиям (что должен сделать определенный пользователь). Выполнение задачи приводит к продвижению бизнес-процесса от одной точки к другой.

В нотации BPMN различают зоны ответственности: пулы и дорожки - графические элементы, служащие для логической группировки операций процесса [2, с. 112]. В карте маршрута $1 \mathrm{C}$ отсутствуют дорожки, но ролевая модель доступна за счет наличия системы адресации бизнес-процессов. Данные, используемые системой адресации, хранятся в регистре сведений. Привязка системы адресации (регистра сведений) к бизнес-процессу осуществляется через объект Задача.

При продвижении бизнес-процесса по карте маршрута в определенных его точках, а именно при переходе на точку действия или вложенный бизнес-процесс, создаются задачи, которые появляются в списках задач соответствующих исполнителей. Выполнение задач осуществляется не только пользователями, но и автоматическими процедурами.

Платформа 1C:Предприятие поддерживает механизм оповещений для объектов конфигурации.

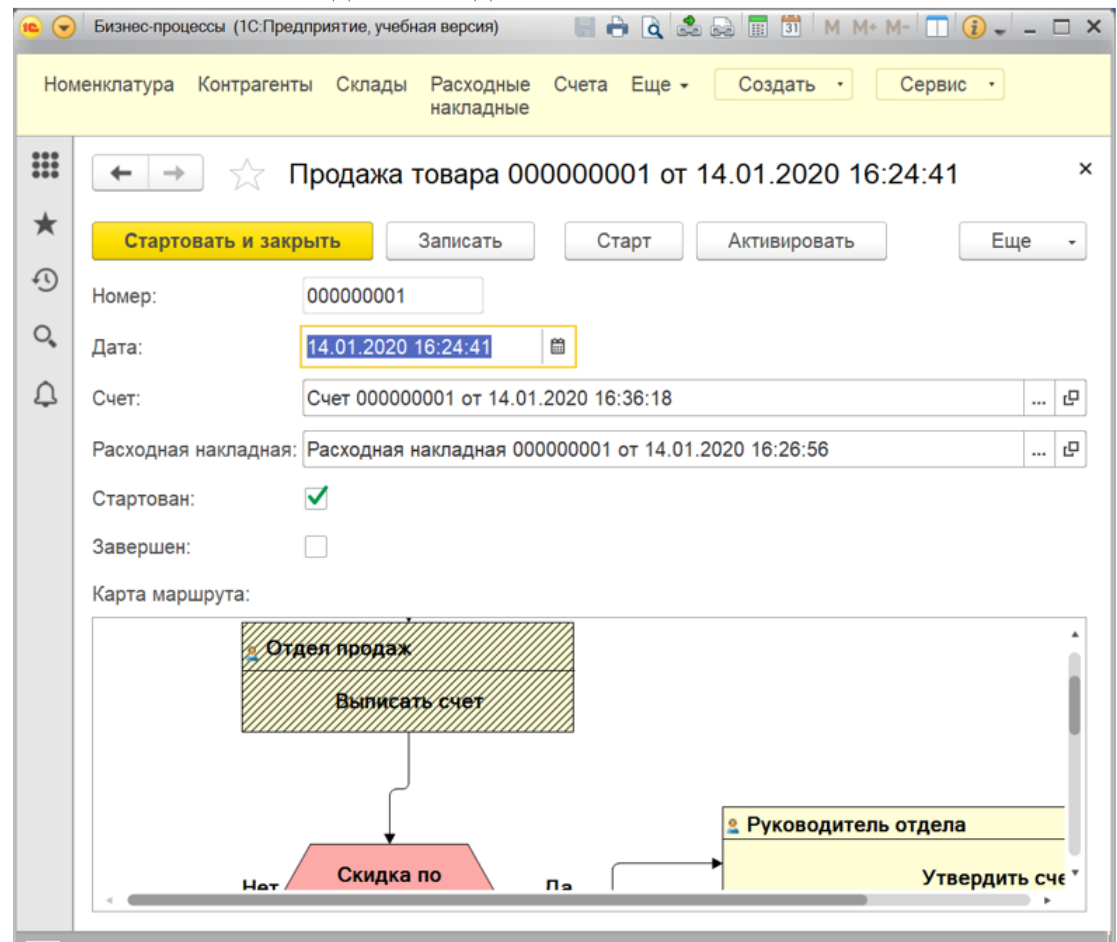

Рис. 2. Механизм оповещений в системе $1 C$

Fig. 2. Mechanism of warnings and reportings in system $1 C$ 


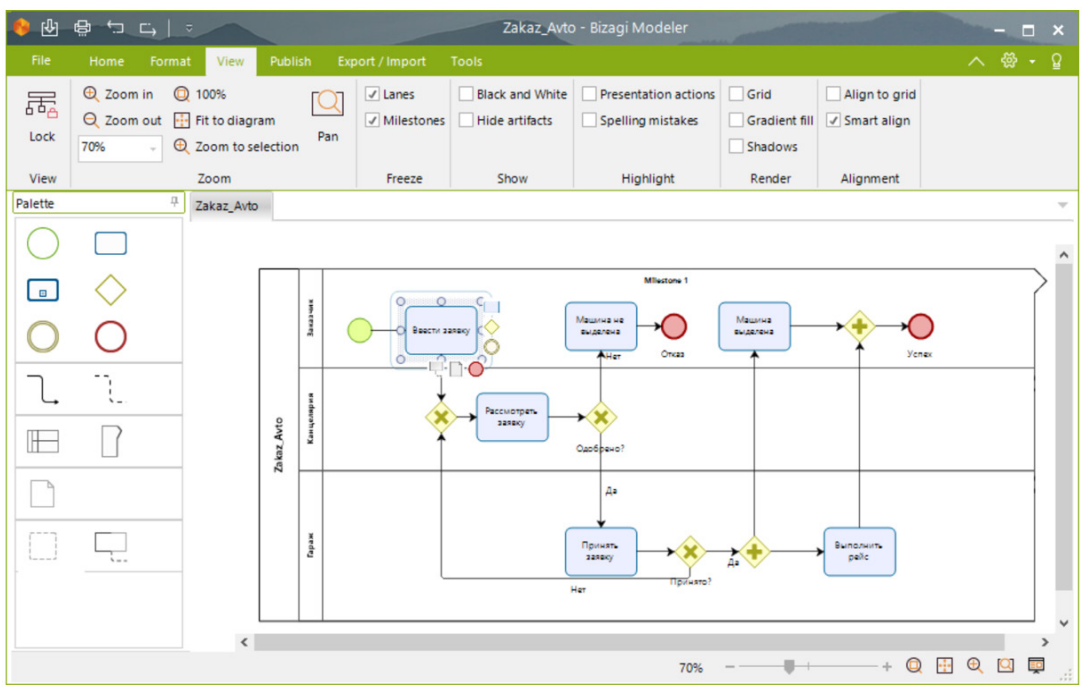

Puc. 3. Модель бизнес-проиесса в Bizagi Studio

Fig. 3. Model of business process in Bizagi Studio

Этот механизм позволяет автоматически открывать формы сопутствующих объектов конфигурации при активации задачи. Например, как видно на рисунке 2 , при активации задачи «Выписать счет» будет автоматически открыта форма нового документа «Продажа товара».

Модель представления данных в $1 C$

Систему BPMS можно рассматривать как среду, где происходит связывание бизнес-процессов с данными. Базовыми понятиями архитектуры любого процессного приложения являются: модель бизнес-процесса, схема данных и формы задач. Моделирование процессов и моделирование данных являются ключевыми задачами разработки.

Модель представления данных - это формализованное представление информационного потока, сопровождающего выполнение процесса. Для системы 1С модель представления данных имеет важную особенность. Платформа 1C поддерживает общую систему типов для встроенного языка, полей баз данных и интерфейса. Другими словами, объекты Бизнес-процесс и Задача являются такими же типами платформы, как и объекты, представляющие данные: Справочник, Документ, Регистр. Разработчик одинаковым образом определяет как поля базы данных, так и переменные встроенного языка, реквизиты форм и одинаковым образом работает с ними [6, т. 1, с. 13]. Разработка модели представления дан- ных для конкретного бизнес-процесса в 1С является процессом простого уточнения: какие данные нужны для его выполнения.

\section{Бизнес-правила в $1 C$}

Реализация бизнес-правил выполняется в элементе Точка условия, который соответствуют элементу Gateways (Шлюз) в нотации BPMN 2.0 [2, c. 90]. В системе 1C «Важной особенностью этой точки является обработчик проверки условия, наличие которого обязательно и контролируется при проверке карты маршрута перед сохранением бизнес-процесса» [6, т. 2, с. 64]. Обработчик проверки может быть предельно формальным, например, параметру СчетУтвержден, со-

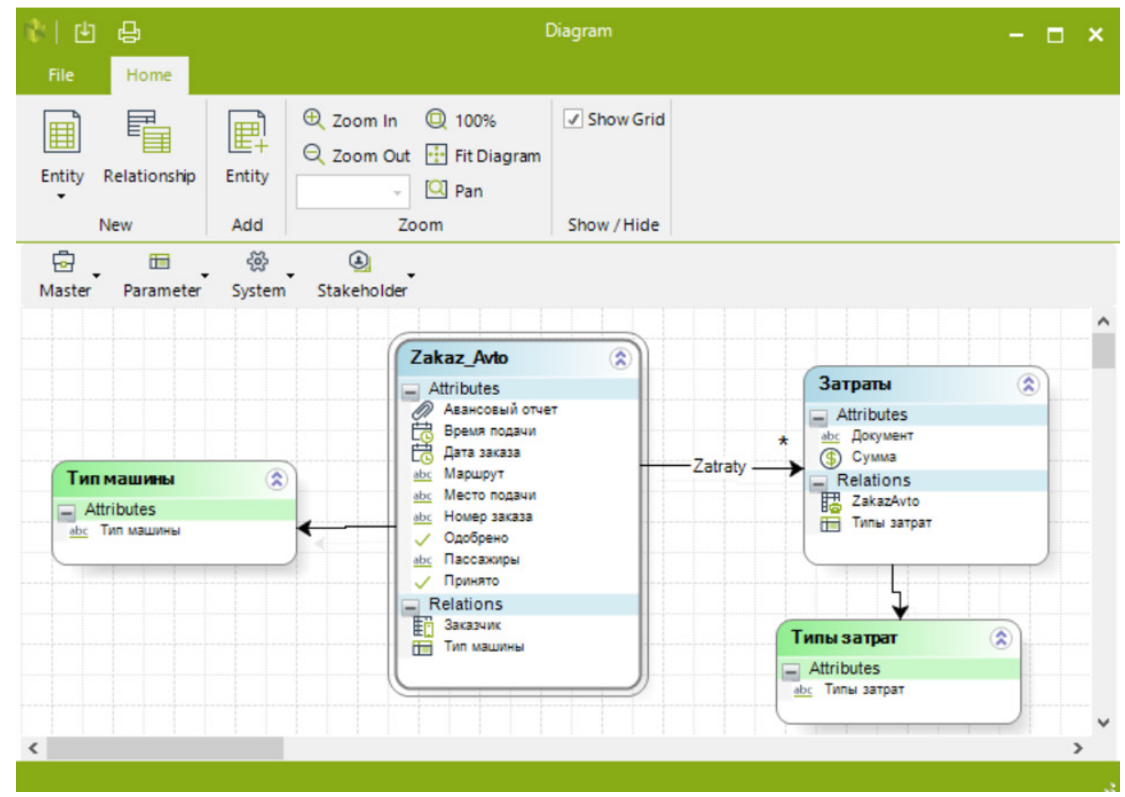

Рис. 4. Логическая модель данных проекта Fig. 4. Logical model of project data стояние которого контролируется, присваивается значение Истина. Обработчик проверки может также реализовывать бизнес-правило, представляющее некоторый алгоритм.

\section{Формык кзадачам \\ бизнес-прочесса в $1 \mathrm{C}$}

Платформа 1C автоматически генерирует форму бизнес-процесса, форму списка, форму выбора, т. е. все необходимые формы для визуализации прикладного объекта Бизнес-процесс. Каждый исполнитель задач бизнес-процесса получит форму списка адресованных ему задач, и каждая задача при ее исполнении будет открыта в стандартной форме - окно с реквизитами бизнес-процесса.

Платформа 1C позволяет описать реквизиты бизнес-процесса посредством ссылочных типов на те документы, которые исполнитель должен использовать для реализации задачи. В обработчике события ПередСтартом можно проверять условия, необходимые для старта бизнес-процесса, создавать «сопутствующие» объекты, ссылки на которые нужно хранить в самом бизнес-процессе. В этом случае у исполнителя открывается не стандартное окно задачи, а тот документ, который требуется для исполнения задачи. 
Разработка и исполнение бизнес-проиессов в системе управления бизнес-процессами Bizagi Studio

Последовательность разработки проекта в Bizagi Studio предполагает использование следующих инструментов:

1. Model process - дизайнер модели бизнес-процесса;

2. Model data - дизайнер логической схемы базы данных;

3. Define form - дизайнер вебформ к задачам бизнес-процесса;

4. Business rules - редактор бизнес-правил;

5. Performers - редактор оргструктуры;

6. Integrate - мастер интеграции со сторонними системами и базами данных;

7. Execute - процессная аналитика и динамическая отчетность.

Построение бизнес-модели также происходит под контролем студии, например, встроенные правила соединения элементов гарантируют соответствие нотации BPMN и формируют подсказки, направляющие работу пользователя как показано на рисунке 3 .

\section{Модель представления данньхх в Bizagi Studio}

Разработчики Bizagi встроили в среду студии полноценный дизайнер реляционной базы данных, функции которого напоминают функции, реализованные в PowerDesigner. Решение получилось очень удачным: система синхронизирует все зависимости между отдельными частями проекта - процессом, моделью данных и пользовательскими интерфейсами. В том числе Bizagi переносит изменения схемы данных из разрабатываемой модели в среду выполнения бизнес-процесса.

На рисунке 4 приведена модель представления данных, выполненная в дизайнере студии Bizagi. Данные, формализованные для представления информационного потока, сопровождающего выполнение процесса, могут быть повторно использованы во всех процессах, созданных в одном проекте без ограничений [7].

Каждый процесс в Bizagi представлен одной сущностью, которая

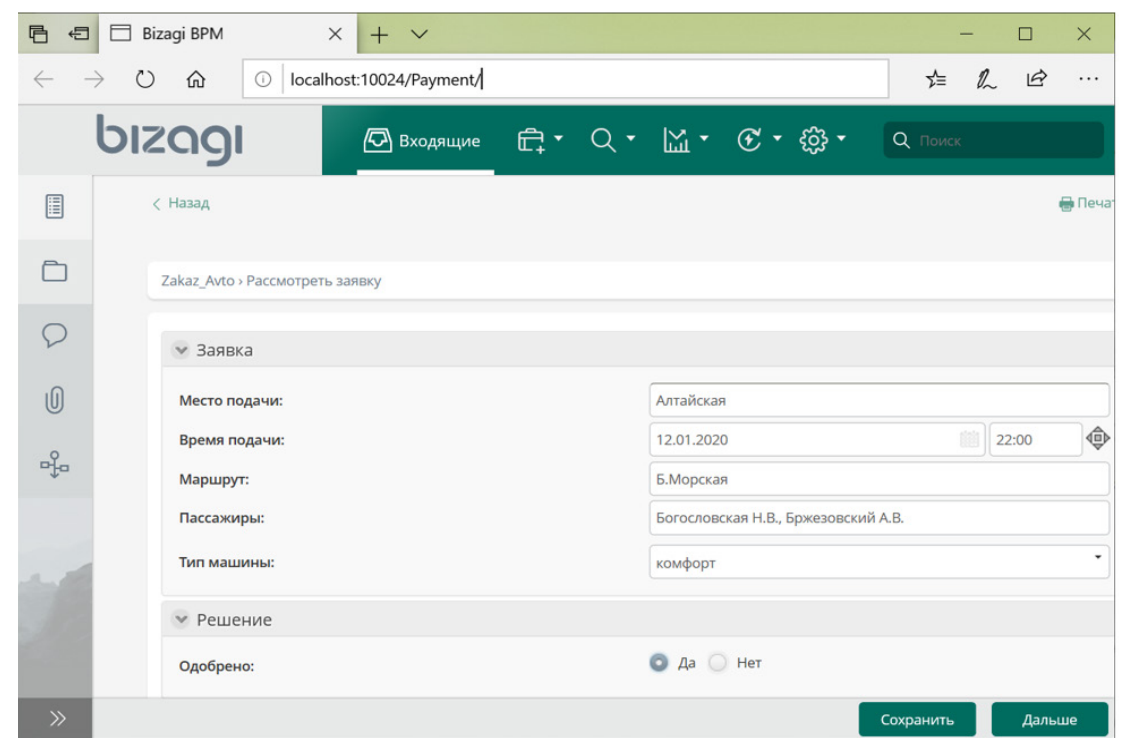

Pис. 5. Исполнение бизнес-процесса в Bizagi Studio

Fig. 5. Execution of business process in Bizagi Studio

обеспечивает входную точку доступа к остальным данным процесса, то есть является основной сущностью, через которую пользователь получает доступ к остальным сущностям модели данных как показано на рисунке 4.

Для обеспечения организованной и согласованной структуры Bizagi предоставляет пять типов сущностей (Master, Parameter, System, Application, Stakeholder) и четыре типа отношений, с помощью которых можно строить модель данных. На рисунке 4 сущности с типом Master изображены синим цветом, сущности с типом Parameter - зеленым. В типе объектов Parameter удобно хранить список предопределенных значений, ввод и редактирование которых возможны во время выполнения процесса.

\section{Бизнес-правила в Bizagi Studio}

Бизнес-процессы регулируются бизнес-правилами, которые обеспечивают их корректное выполнение. Редактор бизнес-правил позволяет создавать, редактировать и удалять бизнес-правила, ориентированные на управление потоком процессов, или выполнять проверки, производить вычисления и т. д.

Бизнес-правила могут быть следующих типов:

- Define expression (определение выражений): определение пути, по которому должен следовать процесс в соответствии с конкретными бизнес-условиями;
- Activity actions (события деятельности): выполнение необходимых процедур при выполнении задачи, таких как проверки и расчеты. Формы к задачам бизнес-проиесса в Bizagi Studio

Конструктор форм управляет всеми пользовательскими интерфейсами для выполнения бизнес-процесса. Разработка формы в Bizagi Studio выполняется перетаскиванием полей данных на форму и упорядочиванием их любым способом, который требует процесс, без необходимости.

На этапе запуска мастер процессов позволяет выполнить:

- развертывание процесса: публикацию процесса в среде исполнения, отличной от среды разработки;

- запуск процесса: тестирование и проверку проекта (рисунок 5).

Заключение (результаты). В статье рассмотрены возможные подходы к созданию и тестированию исполняемых бизнес-процессов нотации BPMN 2.0. Для анализа выбраны система управления бизнес-процессами Bizagi Studio и платформа 1C:Предприятие 8.3 как процессно-ориентированная система автоматизации деятельности предприятий.

Bce этапы жизненного цикла исполняемого бизнес-процесса: моделирование схемы процесса, разработка слоя данных, описание бизнес-правил, регламентирующих процесс, проектирование форм пользователя, запуск и тестирова- 
ние выполняются как в одной, так и в другой системах собственными встроенными средствами.

Разработка модели бизнес-процесса выполняется с помощью панелей элементов, соответствующих элементам нотации BPMN 2.0. В системе 1C количество элементов существенно меньше, но и в Bizagi Studio представлены не все элементы нотации. Большую наглядность моделей в Bizagi обеспечивает применение дорожек, визуализирующих исполнителей. В $1 \mathrm{C}$ система адресации реализована в свойствах задач и отображена в карте процесса в заголовках задач.

Существенные различия анализируемые системы демонстрируют на этапе представления информационного потока, сопровождающего выполнение процесса. Если в 1С общая система типов позволяет довольно простым способом связать данные и задачи бизнес-процесса, то в системе Bizagi разработка модели представления данных требует от пользователя знаний в области БД. Формализация информационного потока, сопровождающего бизнес-процесс, в Bizagi выполняется в полноценном дизайнере реляционной базы данных и требует определять сущности, атрибуты, связи.

Описание сложных, представляющих некоторый алгоритм, бизнес-правил как в 1C, так и в Bizagi требует знакомства с языками программирования.

Построение форм для задач бизнес-процесса в рассмотренных системах выполняется в двух принципиально разных технологиях: в Bizagi - визуального проектирования перетаскиванием атрибутов данных и размещением в форме, в $1 \mathrm{C}$ - технологией метаданных по принципу декларативного описания свойств объектов.

Запуск и тестирование бизнес-процессов как в $1 \mathrm{C}$, так и в Bizagi выполняется в разрезе исполнителей, при этом доступен мониторинг порядка выполнения задач.

\section{Список литературы}

1. Профессиональные стандарты «Специалист по информационным системам» и «Руководитель проектов в области информационных технологий». - М.: ООО «1С-Паблишинг», 2015. - 365 с.

2. Богословская Н.В. О выборе инструмента моделирования BPMN для реализации учебного процесса / Н.В. Богословская, А.В. Бржезовский // Развитие образования. - 2019. - №2 (4). - С. 75-83.

3. Business Process Model and Notation (BPMN) // OMG: Object Management Group. URL: https://www.omg.org/spec/ $\mathrm{BPMN} / 2.0 / \mathrm{PDF}$

4. Bizagi Studio. URL: https://www.bizagi.com/platform/studio

5. Архитектура платформы 1C:Предприятие (версия 8.3.17) [Электронный ресурс]. - Режим доступа: https://v8.1c.ru/ platforma/biznes-processy/

6. Ажеронок В.А. Профессиональная разработка в системе 1С:Предприятие 8: в 2 т. / В.А. Ажеронок, А.П. Габец, Д.И. Гончаров. - 2-е изд. - М.: 1С-Паблишинг, 2012. - ISBN 978-5-9677-1790-8

7. Data Modeling. URL: https://help.Bizagi.com/bpm-suite/en/index.html?modeling_data.htm

\section{References}

1. (2015). Professional'nye standarty "Spetsialist po informatsionnym sistemam" i "Rukovoditel' proektov v oblasti informatsionnykh tekhnologii"., 365. M.: OOO "1C-Publishing".

2. Bogoslovskaia, N. V., \& Brzhezovskii, A. V. (2019). O vybore instrumenta modelirovaniia BPMN dlia realizatsii uchebnogo protsessa. Razvitie obrazovaniia, 2 (4), 75-83.

3. Business Process Model and Notation (BPMN). OMG: Object Management Group. Retrieved from https://www.omg.org/spec/ BPMN/2.0/PDF

4. Bizagi Studio. Retrieved from https://www.bizagi.com/platform/studio

5. Arkhitektura platformy 1C:Predpriiatie (versiia 8.3.17). Retrieved from https://v8.1c.ru/platforma/biznes-processy/

6. Azheronok, V. A., Gabets, A. P., \& Goncharov, D. I. (2012). Professional'naia razrabotka v sisteme 1C:Predpriiatie 8. M.: 1C-Publishing.

7. Data Modeling. Retrieved from https://help.Bizagi.com/bpm-suite/en/index.html?modeling_data.htm

$\quad$ Информация об авторах
Богословская Наталья
Валентиновна - канд. техн. наук,
доцент кафедры информацион-
но-сетевых технологий,
ФГАОУ ВО «Санкт-Петербург-
ский государственный универ-
ситет аэрокосмического прибо-
ростроения», Санкт-Петербург,
Российская Федерация.
Бржезовский Александр
Викторович - канд. техн. наук,
доцент кафедры информацион-
но-сетевых технологий, ФГАОУ
ВО «Санкт-Петербургский госу-
дарственный университет аэро-
космического приборостроения»,
Санкт-Петербург, Российская
Федерация

Информация об авторах Богословская Наталья

Валентиновна - канд, техн, наук, но-сетевых технологий

ФГАОУ ВО «Санкт-Петербургский государственный университет аэрокосмического приборостроения», Санкт-Петербург, Российская Федерация.

Бржезовский Александр но-сетевых технологий, ФГАОУ ВО «Санкт-Петербургский госукосмического приборостроения», Федерация

\begin{abstract}
Information about the authors
Natalia V. Bogoslovskaya candidate of technical sciences, associate professor, Department of Information and Network Technologies, FSAEI of HE "Saint Petersburg State University of Aerospace Instrumentation", Saint Petersburg, Russian Federation
\end{abstract}

\section{Aleksandr V. Brzhezovskii -} candidate of technical sciences, associate professor, Department of Information and Network Technologies, FSAEI of HE "Saint Petersburg State University of Aerospace Instrumentation", Saint Petersburg, Russian Federation.

\section{Авторсем суинчен пёлтерни}

\section{Богославская Наталья}

Валентиновна - техника ӑслӑлӑхӗн к-чӗ, АВ ФПА «Санкт-Петербургӑн аэрокосмос приборостроенийён патшалӑх университечё» $\mathrm{B} \mathrm{H}_{\mathrm{H}}$ информаципе тытӑм технологийӗ кафедрин доценчё, СанктПетербург, Рас̧с̧ей Патшалӑхе̌.

\section{Бржезовский Александр}

Викторович - техника ӑслӑлӑхӗн к-чӗ, АВ ФПА «Санкт-Петербургӑн аэрокосмос приборостроенийӗн патшалӑх университечё» $\mathrm{BУ}_{\mathrm{H}}$ информаципе тытӑм технологийӗ кафедрин доценчӗ, СанктПетербург, Рас̧с̧ей Патшалӑхе̌. 